\section{$132 / 199$ \\ 9 1) LA-7954-MS \\ Informal Report}

Preliminary Considerations Concerning

Neutral Plasma Beam Propagation Acrnss a Magnetic Field 


\title{
Preliminary Considerations Concerning Neutral Plasma Beam Propagation Across a Magnetic Field
}

\author{
William R. Shanahan \\ Rickey J. Faehi \\ Brendan B. Godfrey
}

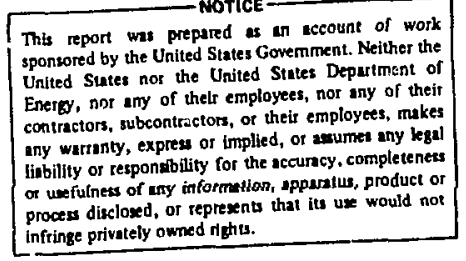




\title{
PRELIMINARY CONSIDERATIONS CONCERNING NEUTRAL PLASMA BEAM PROPAGATION ACROSS A MACNETIC FIEID
}

\author{
by \\ William R. Shanahan, Rickey J. Jaehl, and Brendan B. Godfrey
}

\begin{abstract}
A plan to address physical questions of interest for exoatmospheric military applications of intense neutralized plasma beams is described. After a brief review of earlier work relevant to this; matter and a detailed explanation of why such work cannot answer questions of present interest, we suggest a plan employing interactive application of several numerical and anaiytic techniques to treat relevant phenomena occurring on the various rather disparate time and length scales involved. The first part of the study wonld determine the macroscopic features of beam propagation through calculations effect.ed with a magnetohydrodynamical numerical code. Classical transport coefficients would be employed in this initial phase. Using information thus gained concerning gross charge and current djstributions, we would initialize particle-in-cell simulations to study those microscopic, phase-space-dependent phenomena which can alter the phenomonological transport coefficients appearing in the fluid description. Insight thereby gained concerning anomalous, collectively induced transport effects would then be applied to yield a refined, accurate description of the macroscopic aspects of neutral plasma beam propagation. Personnel and computational resources available at the Los Alamos Scientific Laboratory are described. Results of a very preliminary particle-in-cell simulation of a neutral plasma beam propagating across a magnetic field are presented.
\end{abstract}

\section{I . INTRODUCTION}

Advances in particle accelerator technology offer the possibility within the foreseeable future of producing plasma beans of sufficiently high energy flux and low emittance to be of interest for exoatmospheric military applications. Practical employment of these beams for such purposes depends, however, upon the degree to which they may be propagated over significant distances across the geomagnetic field through the tenuous gas of the upper atmosphere 
without degradation of desired directionality and energy ilux properties. Beam deflection, heating, dispersal, and stability form the principal areas to be addressed. The parameter regimes likely to be of interest indicate that plasma collective effects will play a dominant role. Plasma theory expertise and computational tools availatle at the Los Alamos Scientific Laboratory (LASL) can make a significant contribution to questions concerning neutral plasma propagation in environments of present interesit.

The report is organized as follows: Section II briefly reviews earlier work thought to be relevant to the problem of plasmoid propagation. Such a review serves a number of purposes. Firstly, it indicates the core of results upon which one may draw for initial insight into the problems presented by plasmoid propagation. Secondly, it provides an opportunity to describe some basic results necessary to an intelligent discussion of the issues involved. Lastly, and perhaps most importantly, it serves to motivate the suggested study by indicating why previous work cannot be applied to answer questions of present interest. Section III describes some of these limitations and deficiencies in greater detail. Section IV is a description of a plan of study designed to address these questions. The time-dependent and nonlinear nature of many of the phenomena expected to be relevant indicates that a large part of the study wili be numerical in nature. The plan described envisions the interactive application of several different computational tools to treat phenomena on the rather disparate time and length scales involved. Section $V$ briefly describes the numerical tools to be employed. These tools are for the most part presently available at LASL for immediate use without the need of extensive code development. Indeed, one of the guiding limitations employed in the design of the recommended program of study was that no investigations be included included which could not be addressed with immediateiy available numerical codes. An exception to this limitation appears in the guise of a three-dimensional version of the particle-in-cell (PIC) code CCUBE, which is to be developed for a separate program being pursued at LASL but which will be automatically available for other applications. CCUBE presently exists in a highly versatile two-dimensional version and forms the principal numerical tool employed in investigations of intense particle beams at LASL. Finally, Section VI presents some preliminary results of plasmoid propagation studies made with this two-dimensional version of CCUBE. 


\section{REVIEW OF PREVIOUS WORK}

Propagation of neutral plasma across a magnetic field has previously been discussed in connection with a variety of applications, ranging from the interaction of the solar wind with the geomagnetic field to the injection of plasma into devices designed to achieve thermonuclear fusion. Perhaps the earliest extersive discussion of the problems involved is that of Chapman and Ferraro, 1 given in 1931. These authors, concerned with the origin of geomagnetic storms, postulated the existence of charged corpuscular radiation from the sun, and attempted to deduce some of the consequences. They clearly recognized the essentially collective nature of cross field-plasma propagation. A single particle injected into a magnetic field will execute cyclotron motion and thus fail to penetrate more than one or two Larmor radii. Hence, effective plasma propagation across a magnetic field is dependent upon the departure from single particle motion induced by plasma charges and currents. Chapman and Ferraro recognized essentially two extreme configurations in which plasma propagation can occur. In the first, the magnetic field completely permeates the plasma, leading, via the Lorentz force, to a macroscopic separation of charge. $A$ geometrically finite element of plasma will thus become polarized, with a corresponding electric field existing throughout the plasma in a direction at right angle to the ambient magnetic field. Propagation can then occur unimpeded as an $\mathrm{E} \times \mathrm{B}$ motion. This was the mechanism discussed most extensively by these authors, apparently because they believed this to be the ultimate steadystate established by plasma propagating in a direction other than that parallel to the magnetic field. That a sufficiently dense and fast plasma could also propagate by effectively pushing the magnetic field aside was also pointed out by these investigators. Physically, this arises from the magnetic screening effect $o_{i}$ diamagnetic currents induced near the surface of the neutral plasma, producing a magnetic field free region inside and slightly increasing the ambient field immediately outside. This possibility was mentioned by Chapman and Ferraro in relation to plasma propagation in an inhomogenous magnetic field, but no extensive discussion was presented. They did, however, stress the importance and difficulty of the, presumably analytically intractable, full dynamical problem, a circumstance which perhaps explains their emphasis on the presumed steady-state.

For the next three decades interest in the interaction of streaming plasmas with magnetic fields was virtually confined to those cuncerned with 
geomagnetic phenomena. 2 A particularly relevant contribution from this era was that of Ferraro, ${ }^{3}$ who addressed himself widely to the case of a plasma stream with sufficient kinetic energy density to exclude the ambient magnetic field, as described above. Ferraro was able to provide at least a rough quantitative basis for the qualitative description referred to there. A number of results are of particular interest. Ferraro was able to demonstrate that the magnetic field decayed within a distance of $c / w_{p}$ of the surface of the plasma, where " $c$ " is the velocity of light and " $w_{p}$ " is the electron plasma frequency. This quantity, which has since come to be known as the "collisionless skin depth" is for the solar wind on the order of kilometers, but for the much denser plasmas likely to be of interest for military applications, it is approximately a centimeter. Ferraro also demonstrated that "overtaking" of charge sheets occurs, and, in particular, the surface layer is continually being replenished from the interior of the plasma. It was also shown in this work that inclusion of a realistic mass ratio for the electrons and positive ions led to a significant polarization layer at the surface of the plasma, with an accompanying perpendicular electric field. Inclusion of the effect of magnetic field inhomogeneity led to a retardation effect, of significance for geophysical plasmas but probably of no concern for parameter regimes of present interest.

A further useful result of Ferraro's work is the derivation of a rough criteria indicating when magnetic field exclusion may be expected to occur. This may be stated as: (plasma kinetic energy density)/(magnetic field energy density) $\gg 1$. This result is easy to understand physically. If the magnetic field be regarded as a fluid, then its energy density is simply the pressure of the fluid. Similarly, if the plasma be regarded as a jet of fluid, its kinetic energy density may be interpreted as the corresponding stagnation pressure. The condition that the above ratio be large is then just that the jet be able to penetrate the surrounding fluid. For magnetic field strengths and plasma densities and velocities relevant to military applications, this ratio is in fact quite large, $\cong 10^{10}$. This is a convenient parameter to remember when attempting to judge whether a given piece of experimental or theoretical work is of interest for present concerns.

The advent of efforts to produce controlled thermonuclear reactions generated further interest in the physics of neutral plasma propagation across a magnetic field. Attention here is focused upon the efficient injection of 
plasma irsto a magnetic field configuratior where it may be effectively contained ard heated. Early experiments ${ }^{4}$ on plasma motion along a curved magnetic field led Schmidt ${ }^{5}$ to attempt a self-consistent treatment of plasma motion in a magnetic field on the basis of the guiding center description of a plasma. The experiments considered by Schmidt were conducted in a parameter regime in which. the magneric field could readily interpenetrate the plasma, propagation occuring through $\mathrm{E} \times \mathrm{B}$ motion, and emphasis is placed in his work on such cases. However, Schmidt noted the possibility of diamagnetic effects, and independently derived the above condition for such effects to be dominant.

Baker and Hammel ${ }^{6}$ performed a series of elegant experiments in which the $E \times B$ nature of the motion of a plasma stream across a magnetic field was clearly demonstrated. Using a Marshall gun, these investigators injected a neutral plasma of density $\sim 3 \times 10^{13} \mathrm{~cm}^{-3}$ and velocity $\sim 4 \times 10^{7} \mathrm{~cm} / \mathrm{sec}$ across the magnetic field produced by a pair of mirror coils. A rapid field-plasma intermixing was observed to take place, the magnetic field being little perturbed, as revealed by direct probe measurements. Voltage measurements across the stream demonstrated that an electric field of appropriate magnitude had been established within the plasma to permit an $\mathrm{E} \times \mathrm{B}$ drift motion. Further, plasma motion across the field could be stopped by allowing the field lines to pass througle a conductor, thus draining the polarization charge from the plasma stream. Penetration of fields up to $1.0 \mathrm{kG}$ was observed. Neutral plasma propaga'ion by stream polarization seems thus to have been clearly established for this parameter regime. The experiments by Baker and Hammel were the first of a long series, conducted by numerous investigators, of plasma propagation experiments in which $\mathrm{E} \times \mathrm{B}$ motion played the dominant role. These experiments have been summarized by Komori, et al. 7

A quite separate, and very interesting, development was initiated by Bostick. 8,9 The experiments referred to above generally employed plasma streams of up to a meter in length. Using button plasma sources, Bostick was able to produce small, toroidial bunches of plasma which maintained their identity for up to thirty seconds. These experiments are of interest for two reasons. Firstly, they throw additional light on the matter of cross-field propacation. Bostick was able to detect an electric field associated with these systems roughly equal to that expected from $E \times B$ considerations. A small diamagnetic effect was also observed to be present, although this was much less certain. Secondly, Bostick speculated that the integrity of such 
systems was maintained by a self-consistent interaction arising from currents flowing in the toroids and the resultant magnetic fields. In fact, Bostick coined the term plasmoid to denote such a plasma-magnetic field entity, although we shall continue to use it in its more widely accepted sense àenoting a relatively compact parcel of plasma. That a plasma cannot contain itself indefinitely through electromagnetic fields is indicated by an appropriate form of the virial theorem. ${ }^{10}$ This point has been emphasized particularly by Schmidt $^{11}$ in the present context. However, the virial theorem does not prohibit the retardation of plasmoid disassembly by an appropriately induced current, a point noted and briefly investigated by Shersby-Harvie ${ }^{12}$ in connection with radio-frequency plasma confinement schemes. Whether plasmoid propagation can be significantly enhanced for distances and times of military interest by such means is a question which remains to be investigated.

Recent interest in propagation of a neutral plasma across a magnetic field has focused both on the possibility of using neutralized high-energy ion beams to heat a thermonuclear plasma and on its relevance to possible military applications. Ott and Mannheimer ${ }^{13}$ have examined the former problem. They demonstr te that a neutral polarized plasma moving across a magnetic field is to be expected to expand along the magnetic field, a fact which is easily seen by transforming to the frame of the plasma and noting the existence of a radial electric field immediately outside the plasma. This circumstance was also previously noted by Chapman and Ferraro. ${ }^{1}$ ott and Mannheimer also exhibited a self-consistent equilibrium which excluded the ambient field from an infinitely long beam. This equilibrium is a slight generalization to cylindrical geometry of a calculation presented by $\mathrm{Grad}^{14}$ in his rather elaborate discussion of the boundary layer between a diamagentic plasma and its confining magnetic field. Thompson and Jorna ${ }^{15}$ have addressed analytically several questions relating to beam stability, without, however, probing the nonlinear state of the relevant instabilities, or relating their wor:- back to the macroscopic behavior of the plasmoid. Finally, a report ${ }^{16}$ recently prepared by CALSPAN, Inc. for the Air Force Weapons Laboratory (AFWL) purports to address questions of interest for plasmoid propagation in both laboratory and exoatmospheric parametcr regimes. Some aspects of this report will be discussed further below. 


\section{MOTIVATION OF RECOMMENDED STUDY}

This brief review of earlier work indicates that neutral plasma propagation across a magnetic field is hardly a virgin area of investigation, having been the object of both theoretical and experimental research. One might, therefore, be tempted to conclude that further study is unnecessary, and that answers to present questions of incerest may be obtained by judicious examination of the existing body of results. The present authors believe, for a variety of reasons, that this is not the case. Firstly, and most obviously, previous work has beer concerned with parameter regimes drastically different from those of potential military interest. This is indicated by the accompanying Fig. 1, in which the parameter described above as measuring the importance of diamagnetic effects is given for three different plasma systems. The figure for magnetic confinement experiments is a rough average of those drawn from several sources. ${ }^{7}$ The figure for military applications was calculated on the basis of a recent TRW, Inc. ${ }^{17}$ proposal employing a value of the external field of 0.35 gauss. The small value of this parameter in the case of magnetic confinement related experiments is easy to understand, since one is here concerned with rather strong magnetic fields $(\sim 1 \mathrm{kG})$ and with the relatively modest velocities $\left(\sim 10^{7} \mathrm{~cm} / \mathrm{sec}\right)$ obtainable with conventional plasma guns. Thus, these experiments tend to be dominated by plasmoid polarization effects. On the other hand, for parameters of military interest, at least the early stages of propagation will be dominated by diagmagnetic exclusion of the ambient field.

Another deficiency of earlier work may be exhibited by briefly describing the contents of the recent AFWL report ${ }^{16}$ dealing with cross-field plasmoid propagation. This report recognizes the essentially collective nature of the questions involved and attempts to address them by presenting a catalog of possible instabilities and computing, employing well-known formulae, corresponding linear growth rates. There is something intrinsically unsatisfying with such an approach. Presented with a nonequilibrium plasma immersed in an external magnetic field, one can imagine a bewildering variety of possibly relevant plasma instabilities. What is needed is some selection principle which will permit one to narrow the field of possibilities to those which can be expected to come into play in a major fashion during plasmoid propagation. Fonulation and implementation of such a selection principle should be the first step in any study of plasmoid propagation. 
Further, a simple calculation of linear growth rates does not provide the sort of information which is required. Such a computation does not effectively indicate which instability will play a dominant role, for an instability with a large linear growth rate may well saturate at a low level. Nor does a linear calculation provide that information which is desired for those collective effects wich to play an important role. What is required is knowledge of how the relevant instabilities affect the macroscopic behavior of the plasmoid, primarily through alteration of the transport coefficients. Thus, for example, some heating of the plasmoid is to be expected from the Ohmic losses suffered by the diamagnetic currents induced in the plasma. Computation of such heating on the basis of clissical, discrete paiticle transport is not sufficient, for the instabilities induced by the dianagnetic currents themselves are expected to have a profound effect on the plasma resistivity. Such effects can be addressed only through a completely self-consistent, fully nonlinear calcula* tion.

A final, somewhat more general limitation found in earlier theoretical work in relation to questions of present interest is its restriction to static, geometrically highly idealized configurations. Although Ferraro, ${ }^{3}$ for example, discussed the highly interesting case of diamagnetic effect-dominated plasmafield interaction, he dealt solely with the analytically tractable case of a semi-infinite plasma. In a plasmoid jikely to be of interest for weapons applications, however, finite geometry efte.ts will probably be of central importance. Thus, although the surface diamagnetic current considered by Ferraro could be considered to flow essentially unhindered in the infinite field-plasma interface, in an actual finite plasmoid it will encounter a boundary. The effect of such a boundary on current flow patterns and the resultant magnetic field are difficult to evaluate a priori, but it is unlikely to be negligible. Again, the esentially time-independent nature of previous studies make them unsuitable for present applications. Assumption of an asymptotic, steady-state condition underlies the work of Chapman and Ferraro, ${ }^{1}$ much of the work of Ferraro, ${ }^{3}$ and the linear stability analysis of Thompson and Jorna. 15 In the last case, for example, this corresponds to the selection of an appropriate equilibrium about which to perturb. Such a selection, particularly if it is made for a time late in the flight of the plasmoid, may not correspond to the actual specified initial conditions. 
These considerations may be somewhat clarified by tracing, in \&lightly more detail, the probable sequence of events in the flight of a plaswoid. By the above kinetic energy density consideratiors, one expects the early part of the flight to be characterized by field exclusion from the interior of the plasmoid. This exclusion is produced by diamagnetic currents circulating in a thin surface layer. These currents will eventually decay, certainly due to

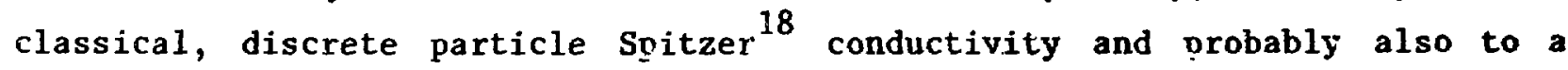
greatly enhanced resistivity due to nunlinear effects induced by instabilities driven by the diamagnetic currents. Thus, the magnetic field will eventually at least partially diffuse into the plasmoid, presumably accompanied by some polarization. A crude estimate for the time for this co occ' $r$ is provided by the well-known formula: ${ }^{18} t_{d} \cong 4 \pi \sigma L^{2} / c^{2}$, where $\sigma$ is the plasmoid conductivity, and $L$ is a scale length. For a plasmoid with $n_{p} \sim 10^{10} \mathrm{~cm}^{3}$ and $T_{e} \sim 1 \mathrm{ev}$, $\nexists d d$ assuming Spitzer conductivity, one obtains $t_{d} \sim 1 \mathrm{msc}$. This is but a fraction of expected times-of-flight, and will be much reduced if nonlinear effects in fact significantly enhance the resistivity. The magnetic field will, therefore, at Least partially permeate the plasma throughout much of its flight. This circumstance perhaps explains the apparently widespread belief that an appropriate model for plasmoid propagation in these parameter regimes is provided by a polarized plasma propagating through $\mathrm{E} \times \mathrm{B}$ motion. Great care is required before such a conclusion cart be properly reached, however. Penetration of the ambient field is a highly dynamic process, and the actual motion is probably determined by a subtle combination of diamagnetic and polarization effects. A model employing a polarized plasmoid would, for example, ignore the strong heating which may occur during the diarnagnetic phase of propagation. An approach based upon a sim : static model as a starting point will always be haunted by doubts concernins the proper choice of initial conditions.

A plan of study of plasmoid propagation must, therefore, incorporate several features if it is to provide answers to questions of practical interest. Firstly, it must provide a methodology through which an intelligent selection from the multitude of possibly relevant collective effects can be made. The criteria of relevance is that of whether the collective effect in question can exert a significant effect on the macroscopic behavior of the plasmoid. Otherwise, the study is likely to degenerate into a listing of amusing plasma instabilities. Secondly, the relevent collective effects must 
be investigated by a method which can effectively address time-dependent, nonlinear pheriomena in realistic geometries. Such an investigation is generally impervious to complete analytic treatment and must, therefore, be designed to be primarily numerical in nature. Thirdly, the enhanced understanding of microscopic collective effects must ;e employed to yield a refined treatment of the macroscopic behavior of the plasmoid. Lastly, an overall objective of any theoretical investigation should be the design of a practical, scalable experiment to verify the principal aspects of the theory. We believe the plan of study described in the next section embraces these features.

\section{PLAN OF RECOMMENDED STUDY}

Plasmoid propagation is one of a large class of problems in which answers to questions of practical concern are determined by interaction between physical phenomena occurring on a number of rather disparate length and time scales. In the present case, for example, inicroscopic collective effects must be examined on a time scale roughly determined by the reciprocal of the electron plasma frequency, which for typical parameters is approximately a tenth of a nanosecond, while total times of flight are expected to be several milliseconds. Such problems are usually impervious to treatment by a single analytic or numerical technique. Generally, an interactive approach employing several techniques is required. In this section, we describe the principal components of such an interactive approach designed to answer key questions concerning plasmoid propagation across a magnetic field.

The general scheme upon which our plan of study rests envisions the application of heuristic, macroscopic considerations and computations to provide initializing data with which to perform detailed PIC simulation of microscop:c, velocity-space dependent collective phenomina. The results of such simulations will then be employed to yield a refined description of parameters which enter into the macroscopic treatment, thus leading to an improved description of plasmoid propagation. This approach is depicted schematically in Fig. 2. The numerical means with which to execute this plan are similarly depicted in Fig. 3. In this section, we shall refer to the numerical methods only in the broadest terms, deferring to section $V$ the cataloging and description of the numerical tools actually available. Figs. 2 and 3 should be regarded as suggestive, rather than as definitive. In particular, the arrangenent of goals and means illustrated there is meant to be more conceptual than chronological. 
Although beginning at the apex of the triangle plan and proceeding in the directions indicated by the arrows does provide a rough description of the anticipated flow of work, it does not completely portray the richly interactive nature of the proposed study. Thus, heuristic and microscopic considerations will probably enter at all stages, and not be confined to easily identified segments of the study. The reinforcing flow of information expected to characterize this study is depicted in Fig. 4. In the remainder of this section, we shall explain these diagrams in detail.

As emphasized in Section III, any study of plasmoid propagation should begin by selecting from the welter of collective effects those which play a dominant role. Of course, such a selection must itself be part of the initial phase of the study, and not be made on the basis of preconceptions. Apart from simple physical arguments, such as those made in Section II regarding the importance of diamagnetic as compared to plasmoid polarization effects, this choice of collective effects for detailed microscopic simulation will probably be most effectively made by employing a variety of computational tools. A logical first step, as indicated in Fig. 2, involves the determination of the gross features of charge and current distribution. Such a determination may be made with a magnetohydrodynamic, fluid code, several of which are currently available at the Los Alamos Scientific Laboratory. Some of these codes will be described briefly in the next section. These codes employ as their basic dynamical element the macroscopic, fluid equations expressing mass, momentum and energy conservation. No detailed particle dynamics are included. Hence, a number of quantities, which are in reality determined by such dynamics, such as transport coefficients, must be specified before computation commences. It is a refined description of such parameters, and their eventual incorporation into fluid codes, which is a primary goal of the program.

Initially, however, arbitrary but reasonable values for these parameters will be supplied. At least one of the $\operatorname{codes}^{19}$ which is expected to be used automatically selects, without user intervention, the collisional, Spitzer ${ }^{18}$ values for these quantities, and this seems a natural starting point. It is to be emphasized, however, that there is no intrinsic barrier to enhancing or decreasing these parameters, and thus obtaining preliminary insight into the possible effects of collectively induced anomalous effects.

Although it is naturally difficult to completely anticipate the results of such a study, one nevertheless has some idea of the variety of information to 
be obtained. One should be able to examine the diamagnetic currents present in the initial phase of propisation and observe their effect on the ambient field. Further, since the codes incorporate resistive diffusion, the decay of these currents and the accompanying magnetic diffusion inio the plasmoid can be examined. Since fluid codes operate typically on time scales much longer than those relevant to particle-in-cell simulations, we expect to be able to follow the details of magnetic field diffusion and plasmoid polarization for realistic time intervals. The relevant fluid codes self-consistently incorporate heating due to Ohmic losses, and thus the important questions of plasma heating and resultant hydrodynamic expansion may be tentatively addressed. For Spitzer values, these are expected to be insignificant; but, as already noted, one may artificially enhance the values of the relevant transport coefficients, and thus obtain initial, though not self-consistent, information concerning the consequences of collectively enhanced resistance and diffusion.

Information concerning gross charge and current distribution is probably the most important datum to be obtained from these fluid, numerical studies, but it is by no means the only one. Thus, by initializing the plasmoid with an unfavorable configuration, one should be able to obtain at least partial information concerning possible macroscopic fluid instabilities. Should such instabilities prove to be a dominant feature of plasmoid propagation, this would, of course, be a crucial, perhaps determining, piece of information. Methods of possibly enhancing propagation, such as by inducing a current in the plasma, should also be illuminated through a fluid analysis. Finally, it appears feasible to examine a range of plasmoid densities and density profiles.

This last point is one aspect of an issue which deserves to be discussed explicitly. Although a particular weapon scheme or experiment may be confined to a relatively small parameter regine, a comprehensive theoretical investigation should address itself to as wide a range as is practical. Thus, although magnetic field strengths in expected environments of operation are only a small fraction of a gauss, we intend parameter studies which enzompass considerably larger fields. Again, though plasmoid densities of several $10^{10} / \mathrm{cm}^{3}$ have been mentioned, and are used several times throughout this report for iilustrative purposes, other density regimes should be examined. As a final example, we mention the effect of a background gas. The measures to be taken to address this question will be described below, but we wish to emphasize nere that 
studies in neutral density ranges other than that expected in regines of ultimate operation $\left(10^{10} / \mathrm{cm}^{3}\right)$ should be investigated.

The considerations which make such a wide parameter range desirable are two-fold. First, it generally increases the utility of the study. New regines of possible applicability may emerge. The second, and more practical, consideration is that an important goal of any theoretical study of plasmoid propagation should be the design of a practical, scalable expcriment. Obviously, experiments on the length and time scales appropriate to envisioned applications are not practical. It, therefore, becomes imperative to detennine the scaling of relevant plasmoid propagation characteristics with respect to the several parameters involved, particularly those that may be easily varied experimentally. For example, the effect of the weak geomagnetic field is of significance primarily due to the large distances over which the plasmoid is expected to propagate. It is, therefore, possible that the unrealistically short distances encourtered in the laboratory can be compensated by a corresponding increase in the magnitude of the magnetic field. A theoretical study should reveal the appropriate scalings, an objective achievable only by as extensive a parameter study as possible, consistent with time and computational constraints.

Returning briefly to the matter of fluid, macroscopic calculations, it is perhaps well to emphasize that although such calculations cál yield extensive and crucial information, they possess certain intrinsic limitations. Quite apart from the obvious fact that such calculations cannot address questions related to detailed particle dynamics, there are a number of restrictions which arise essentially from the two-dimensional character of the codes to be used. Although a three-dimensional version of one of the codes to be described in the next section does exist, the coarse zoning employed renders it of little use. An example of a restriction of some possible consequence appears in an Eulerian magnetohydrodynamic code ${ }^{19}$ which is currently being considered for use in the suggested study. In this code, all of the fluid motions are confined to a plane, while the magnetic field is pointing perpendicularly to the plane. For many aspects of plasmoid propagation, such a configuration is ideal. One may thus, for example, examine the polarization charge expected to be present during late stages of propagation. On the other hand, as shown by Chapman and Ferraro ${ }^{1}$ and 0 tt and Manheimer ${ }^{13}$ such a polarized entitly will experience. electromagnetic forces that will lead to enhanced diffusion of plasma along the 
magnetic field lines. Since fluid motion is confined to the plane, this possibly important aspect of propagation cannot be addressed with such a numerical tool. Further, certain features of possible confinement schemes employing induced currents seem to be intrinsically three-dimensional. These observations are not meant to suggest that the two-dimensionality of these numerical tools precludes their useful application to plasmoid propagation studies, but only to emphasize that a variety of analytic and numerical techniques must be employed to yield a complete picture. Indeed, some of the further aspects of the heuristic phase of our study, now to be described, at least partially address these questions.

An examination of Fig. 3 reveals that particle-in-cell simulation techniques play a role in both the heuristic and microscopic parts of our scheme. That they appear in the microscopic part is not surprising, for such techniques as: designed specifically to examine particle, velocity space-depend at phenomena. Their incorporation in the heuristic component requires additional explanation, however. Such incorporation is made possible by the extreme versatility of particle-in-cell computational methods, particularly as they appear in the code CCIjBE, developed by B. Godfrey. ${ }^{20}$ CCUBE is a two-dimensional, electromagnetic, fully relativistic particle-in-cell ${ }^{21}$ simulation code capable of treating any orthogonal curvilinear coordinate system. In the past CCUBE has been used primarily to investigate the highly nonlinear, phase spacedependent phenomena so characteristic of plasma physics. This will be its principal role in the present study as well. However, it is possible to employ CCUBE in a mode which yields daca more akin to that derived from fluid calculations than that generally associated with particle-in-cell simulations. For example, one may simulate the injection of a neutral plasmoid into a region filled with transverse magnetic field and observe the resultant field exclusion and accompanying dianagnetic currents. In fact, a simulation of this variety has already been performed to verify our qualitative picture of the very early phases of propagation. A report of the principal results of this simulation forms the bulk of Section VI. Here, we wish merely to indicate some of the general aspects of using CCUBE in this heuristic mode.

By employing CCUBE in the heuristic mode, we mean quite generally any application in which the plasmoid is simulated as a whole, rather than one in which some particular region, such as the sheath in which the diamagnetic 
currents flow, is examined. The advantages which accrue by using a particlein-cell code for these purposes, rather than relying solely upon the results of a fluid computation, are several-fold. Firstly, a separate, independent check is established. Although all the codes to be employed have been used previously, enough uncertainties usually enter in the course of a particular application that the establishment of mutual verification is, where possible, a desirable feature. Secondly, since such codes compute the detailed particle motion, examination of phase-space plots and current distribution can yield information on actuai particle paths. Such paths are likely to be rather complicated in the environment of a geometrically finite, propagating plasmoid. As we shall indicate further below, such information is likely to be of more than academic interest. As a final example of such use of CCUBE, we return to the question of enhanced plasma spreading along a magnetic field. As explained earlier, the most useful fluid code available cannot address this question, since fluid notions are confined to a plane while the magnetic field is pointing perpendicularly to that plane. CCUBE does not suffer from this deficiency, since the magnetic field can lie either in the plane of motion or perpendicularly to this plane. A combination of fluid and heuristic CCUBE computations can thus lead to a fairly complete picture of this phenomena.

Further insight into the nature of using CCUBE in the heuristic mode can be gained by noting an operational detail which differs from that in the usual applications. In many microscopic collective phenomena an important role is played by the response of the ions. Simulations must, therefore, be run a sufficiently long time to allow the manifestation of such a response. If one were to use a realistic mass ratio, the resulting simulation would require an intolerably long time with a corresponding computational expense. It. is, therefore, customary to use an unphysical mass ratio which nevertheless preserves a sufficient dynamical difference between electrons and ions. The situation can be significantly different for applications in the heuristic mode of operation. For example, in the simulation to be described in Section VI, our chief concern was with the gross features of field exclusion. Since we have previously indicated that the occurence of such exclusion is controlled by relative sizes of magnetic field and plasmoid kinetic energy densities, it was both possible and desirable, though perhaps not vital, to employ a realistic mass ratio. This example serves to delineate some of the differences between the heuristic and microscopic applications, although it also serves to remind 
us that such distinctions are at least in part arbitrary. If one were interested in the effect of the ion response on the enhanced spreading along field lines discussed above, one would likely employ an artificially small value of the mass ratio.

At present, one factor seriously militates against the use of CCUBE for such heuristic purposes. In the simulation to be described in Section VI, the plasmoid was injected into a region containing a transverse magnetic field. The plasmoid then propagated the length of the computational box, exiting the far side. Thus, the length of time ore could simulate the behavior of the plasmoid was dictated by the time taken to traverse the box. As emphasized at the beginning of this section, it is unrealistic to attempt to simulate the entixe flight of a plasmoid with CCUBE due to the disparate time scales involved. Nevertheless, it appears to be practical to simulate times considerably greater than those $\left(\sim 200 \mathrm{w}_{\mathrm{p}}^{-1}\right)$ dictated by computational box transit times. The desirability of doing so is enhanced when it is recalled that different portions of the flight other than the initial pericd may be thus simulated by initializing CCUBE with parameters taken from fluid calculations. A method of obviating the transit time problem must, therefore, be found and implemented. One obvious way which yields some modest improvement is by the simple expedient of increasing the length of the box. However, this path is clearly blocked by rather definite memory limitations. A much more satisfactory approach consists of transforming the entire computation to the frame of the plasmoid. The plasmoid will then appear to be stationary at the beginning of the simulation and will move about only due to macroscopic forces. It is unlikely that any of these will be sufficiently strong to evict the plasmoid from the box. Implementation of such a plasmoid frame of reference transformation will be the first step in the systematic application of CCUBE in the heuristic mode.

At least two different ways of effecting such a transformation appear possible. In the first, examination of a simulation in which the plasmoid is injected into a magnetic field-filled region may reveal a simple analytical expression for the resultant magnetic field configuration and corresponding plasmoid currents. The transformed quantities, and the accompanying electric fields which result from transformation, may then be used to initialize a simulation in the plasmoid frame. This approach has the virtue of encompassing a range of plasmoid and field parameters without the additional expense of 
running the corresponding injection simulation. However, one is here likely to find it necessary to make approximations to obtain an easily codable initin1izati in procedure. A somewhat more direct, exact approach is to keep the data from a favorable point in an injection simulation, insert a subroutine which effects the appropriate transformations, and employ the result to initialize a plasmoid-frame study, in keeping with our goal of eliminating possible preconceptions. Possibly, a combination of the two will ultimately prove the nost effective.

Having discussed in some detail one aspect of the heuristic phase of the proposed program, we shall pass directly to a description of the principal component of the microscopic portion, momentarily deferring a discussion of the second part of the heuristic study, concerning the noncollective aspect of interaction with a background gas. This is in fact a logical progression, since those heuristic computations described thus far are designed, as indicated in Fig. 4, to provide initializing charge and current distributions for microscopic, particle-in-cell simulations. Although questions of interastion with a background gas are not unrelated, the purposes and methodology of this part of the study are sufficiently different that they are best discussed separately. Since the microscopic simulations are designed primarily to yield refined information concerning the effect of collective phenomena on transport coefficients, we begin with a brief explanation of how such effects can be expected to arise.

Several times in the preceeding discussion, we have referred to anomalous, or collectively enhanced, transport properties, as opposed to those determined by discrete, two-particle interactions. This difference is perhaps most easily exhibited in the case of plasma diffusion across a magnetic field. If twoparticle, or collisional, diffusion predominates, then simple random walk arguments $^{22}$ indicate that the phenomenological diffusion coefficient will be proportional to the square of the random step size. For cross-field diffusion, a logical choice for this quantity is the ion Larmor radius, which is inversely proportional to the strength of the magnetic field. Hence, one expects the cross-field diffusion to scale inversely as the square of the magnetic field. It has been known for some time, however, that magnetic fields are not this effective in suppressing plasma diffusion, the scaling being, for simple geometries, more nearly inversely proportional to the field strength. This is the so-called "Bohm" diffusion regime. 23 A simple, qualitative, explanation 
was first offered by Spitzer. ${ }^{24}$ If rlectric field fluctuations in the plasma, due, for example, to an instability, act perpendicularly to the direction of the magnetic field, then one might intuitively expect the particles to migrate across the field at a rate given by $[\mathrm{E}(\mathrm{t}) \times \mathrm{B}] / \mathrm{B}^{2}$, thus giving rise to the Boinm scaling law. Of course, this rough argument must, for particular applications, be supplemented by a rigorous, self-consistent treatment. Such a treatment was first provided by Rosenbluth and Drummond ${ }^{25}$ for the particular case of an electric field spectrum generated by the ion cyclotron two-stream instability, and this treatment has subsequently been extended and refined. ${ }^{26}$ For present purposes, it suffices to note the dramatic change which collective effects have introduced into diffusive phenomena, changing the scaling from $1 / \mathrm{B}^{2}$ to $1 / \mathrm{B}$. Obviously, an investigation of diffusion which did not include collective effects would lead to highly erroneous results.

Potentially more important for plasmoid propagation matters is the concept. of anomalous resistance. If the electrons in a plasma experience an effective collision frequency $v^{*}$, then the resultant conductivity is given by $\sigma=n e^{2} / \mathrm{m} v^{*}$. In the case of a quiescent plasma in thermal equilibrium, $v *$ is determined by discrete, two-particle effects. The resultant conductivity is the so-called Spitzer, ${ }^{18}$ or collisional, conductivity, which exhibits a distinctive temperature dependence of $\mathrm{T}^{3 / 2}$. It is frequently found, however, that the conductivity of a plasma is considerably lower than that predicted by the Spitzer formula. Such enhanced, or "anomalous", resistivity typically arises when the current flowing in the plasma exceeds some critical value, $j>j_{c}$. Buneman 27 originally suggested that such anomalous resistivity could be due to collisions of electrons with suprathermal collective fluctuations arising from instabilities associated with the current j.tself. One may alternatively view this process as induced wave emission by the electrons, thus leading to an increased effective collision frequency $v *$. The origin and nature of anomalous resistivity has formed one of the principal themes of plasma physics research during the past two decades. Such investigations generally resolve themselves into, first, a search for a possible instability to give rise to the required suprathermal fluctuations, and second, a determination of the nonlinear state of the instability. The electron-ion ${ }^{27}$ and the ion-acoustic 28,29 instabilities have been particularly extensively investigated from this point of view. A recent review by Sagdeev ${ }^{30}$ summarizes previous research in this area. 
For present purposes, the important point to note is that for the mucroscopic, fluid calculations described earlier, one must provide the values of the relevant phenomenalogical transport coefficients which appear in the magnetohydrodynamical equations. Obviously, supplying the incorrect values could lead to highly erroneous results. For example, providing a value of the conductivity derived from the Spitzer formula could lead to a much smaller Joule heating, and ascompanying plasmoid dispersal, than that obtained from more realistic, collectively enhanced resistivity. Sources of such enhanced resistivity are the suprathermal plasma fluctuations arising irom possible current-induced instabilities. The possibility of such instabilities is already revealed by our heuristic-considerations, which indicate that a diamagnetic current will, at least initially, flow in a thin sheath region of the plasmoid. The relevant value of the collectively determined transport coefficients can be determined, however, only by detailed microscopic calculations which effectively incorporate the highly nonlinear effects which characterize instability saturation. Due to the generally analytically intractable nature of such calculations, they are usually effected numerically with a particle-incell simulation code, such as CCUBE. Useful simulations require, in turn, realistic initialization data, to be obtained from the heuristic computations described earlier. This circumstance explains the cyclic nature of the study, as depicted in Fig. 4.

As indicated in our brief discussion of anomalous resistivity, studies of this variety divide themselves into a linear and a nonlinear part. Particlein-cell simulation techniques are essential to the latter phase. In a sense, they also automatically address the linear part as well, since the early phases of instability are presumably determined by linear response. One approach to the complete problem, therefore, is simply to take the initializing data provided by heuristic computations, and perform a PIC simulation of, for example, the sheath region. Such a way of proceeding is, however, generally neither the most efficient nor the most informative. One should presumably make a separate, previous study of the linear theory. There are several reasons for this. Firstly, one wishes to gain some insight into the kinds of instability to be expected. The electron-ion two-stream and ion-acoustic instabilities have been extensively investigated as sources of anomalous resistivity, but it is by no means assured that they will play a dominant role in plasmoid propagation. Indeed, since attention will be focused, at least initially, on the 
current-carrying sheath regions, where large gradients exist, it is possible that velocity-shear driven, Kelvin-Helmholtz-type instabilities ${ }^{31}$ will be of major importance. Secondly, it is frequentiy important to know the frequency range in which a given instability will possess its maximum growth rate. Although in principle a given instability will eventually grow from noise, it is often much more efficient to excite the simulated plasma at the appropriate frequency, thereby giving the relevant mode a "head start." Lastly, a linear analysis will provide threshold data which is important both for initializing the PIC simulation code and for assessing the actual physical relevance of a given instability.

Linear stability analysis has formed one of the principal areas of theoretical plasma physics. ${ }^{32}$ A large body of results exist, and one might hope to glean the required information from a sedulous examination of these results. Unfortunately, this is not likely to prove the case. The reasons are very similar to those adduced for not relying on previous plasmoid studies to answer questions of interest for military applications. Primarily, these are that previous studies are usually in parameter regimes sufficiently different to render their applicability to problems of present interest questionable. Further, these calculations have generally been effected for idealized geometries under various simplifying assumptions. Even those which include some effects arising from the presence of an inhomogeneity ${ }^{33}$ frequently make approximations to render particle orbit analytically tractable. This last point is potentially of some importance here. Although a linear analysis may be made with either a fluid or a kinetic model of the plasma, it is generally preferable to effect, where possible, a kinetic treatment. A kinetic treatment includes such effects as Landau and cyclotron damping and can thus yield thresholds and more accurate growth rates. Further, thermal effects can change the nature of the resultant electronagnetic field structures. Such analyses can be effected only if the nature of particle orbits is clearly understood. Even in the geometrically idealized calculation of sheath structure presented by Grad, ${ }^{14}$ the relevant particle orbits could be quite complicated. For the environment presented by a geometrically finite plasmoid propagating across a magnetic field, this is likely to be even more the case. Obtaining partial insight into this problem was one reason given for running CCUBE in the heuristic mode, and one can now see the practical importance of this exercise. It is unlikely that the required information can be obtained in this fashion alone, 
and further analjtic and numerical work will be required. Precisely what this work will involve is difficult to anticipate, but it may well be similar to the work performed by Speisser ${ }^{34}$ in his investigations of particle motion in geometrically complex magnetic fields. Of course, the relevant orbit computations must then be used to yield linear stability information. This is likely to require some numerical analysis not readily available in existing codes. The programming effort required, however, is anticipated to be considerably less than that involved in the creation of a major computational code. Nevertheless, the analysis of linear stability should be one of the more challenging aspects of the program.

Having performed heuristic fluid and PIC computations and effected a reasonable linear analysis, one is hopefully prepared to perform meaningful, microscopic simulations of those phenomena which give rise to anomalous phenomenological transport coefficients. The information thus far gained will be employed to initialize CCUBE and, perhaps, to excite the appropriate mode. The resulting collective effects are likely to be associated with the nonlinear state of an instability driven by, for example, the diamagnetic currents circulating in the sheath region. Although these nonlinear effects are likely to prove of some scientific interest in their own right, we are, of course, primarily interested in extracting from them a meaningful anomalous resistivity. This quantity would ideally be given by some complete nonlinear theory from spectral information provided by the simulation, but, at least for the cases considered previously, there is a much more practical and direct method of obtaining this information. For current-driven instabilities, one simply applies an external electric field of sufficient magnitude to obtain a constant current. If the current is of sufficient magnitude to initiate instabilities and the accompanying enhanced resistivity, the magnitude of the requisite electric field will increase. Such a procedure has been used, for example, to study the anomalous resistivity induced by the ion-acoustic instability in a uniform plasma. For geometrically more complex situations, it is not clear that this technique would yield meaningful results. It is not immediately clear, for example, if there is an analogous procedure for measuring the anomalous resistivity induced by velocity shear-driven instability.

A number of problems of this sort would likely present themselves during the course of the simulation studies. Their nature and scope are difficult to anticipate at this juncture. However, there is at least one area of potential 
difficulty which is apparent and for which appropriate methods of solution are being sought. As we have indicated, the principal numerical quantity to be evaluated in these simulations is the collectively enhanced, or anomalous, electrical resistivity. In an actual, physical plasma, there is also the classical, Spitzer conductivity arising from discrete, collisional particle effects. In a real plasma in which an instability is generating anomalous resistivity, this classical contribution generally represents a small correction. For a simulated plasma, however, where there is a small number of particles in a Debye "sphere", this is not necessarily the case. Hence, the simulation method introduces numerical resistance, for which one must supply a correction. Ideally, this correction is provided by a detailed analysis of the numerical methods employed. Such analyses have previously been presented for a variety of simulation techniques. ${ }^{35}$ It is not clear, however, that these methods are applicable to the PIC code to be employed in our studies.

It is much more preferable to adopt a direct method of measuring this resistivity. A variety of possibilities suggest themselves. One may, for example, measure the slowing down and stochastic heating of a stable beam of particles propagating through a background plasma and thereby infer a collision rate. Alternatively, one may observe the decay rate of an initially excited plasma mode. There are also analogous methods more closely related to the actual instabilities to be simulated. For example, if threshold data are available from a linear analysis, one may initialize the simulated plasma with parameters in the stable regime and observe the field necessary to maintain a constant current. Since no resistance-producing instabilities would be present, a fact that can be independently verified, the measured resistance would be that due to numerical discreteness effects. Finally, if one is concerned with a slow-growing instability which is not artificially excited initially, there is likely to be an appreciable time during which no effects of the instability will be present. Observation of the required external electric field required to maintain constant-current conditions during this phase of the simulation will, therefore, provide the desired information. This last method is particularly desirable, since one is spared the expense of a separate simulation. The foregoing brief discussion of this particular issue is meant only to indicate the variety of problems to be encountered in the proposed simulation study, and some of the possible paths of solution. 
The microscopically determined collective enhancement of electrical resistivity must now be incorporated into the fluid calculations which determine the long-time, macroscopic behavior of the plasmoid. This is the task indicated on the third heuristic side of our triangular plan, Fig. 2. Since this step occurs at a relatively advanced stage of the study, it is difficult to be very precise concerning the work involved. Presumably, experience and insight gained in earlier phases would play an important role in determining our approach to this -oblem. Basically, as we noted earlier, one may artificially enhance the transport coefficients which enter into the macroscopic, fluid equations which form the dynamical core of the magnetohydrodynamic codes. Whereas this may be done initially in a more or less arbitrary fashion, one wishes to eventually effect this in a manner guided by the microscopic particle-in-cell determination of the relevant transport coefficients. This step should yield a refined, accurate description of the macroscopic behavior of the plasmoid. Of course, the final results of this process should not yield a description drastically different irom the initial heuristic computations, for otherwise the data used to initialize the particle-in-cell simulations would become suspect. Indeed, the entire procedure possesses an iterative nature, a fact which further argues against regarding Figs. 2 and 3 in a strictly chronological sense. It is likely that both heuristic and microscopic computations will be performed throughout most of the course of the study.

A further feature of the third, heuristic phase of our study deserves comment. As indicated in Fig. 2, we hope during this stage to investigate at least some aspects of confinement schemes. For example, one wishes to determine whether the inducing of an initial current in the plasmoid can significantly retard the dispersal due to heating effects. It would seem premature to undertake such studies until it is clear to what extent such dispersal actually occurs. Furthermore, the currents thus induced also have the potential for initiating microinstabilities and accompanying anomalous resistivity. A full understanding of the possible effect of confinement measures is, therefore, possible only after microscopic investigation has revealed the extent and nature of such phenomena in a plasmoid environment. Lastly, many features of confinement schemes, such as actual current paths and resulting confining forces, seem to be intrinsically three dimensional. No computational tool now available can completely address such questions. It is possible that, during the latter stages of the proposed study, a fully three-dimensional version of 
CCUBE will he available. Although this is not an assured fact, such a code would permit one to at least tentatively answer many questions about which one may presently only speculate on the basis of extrapolations of two-dimensional results. For all these reasons, we would reserve to a time relatively late in the study investigation of confinement matters.

Thus far in our description of the suggested plan of study of plasmoid propagation, we have only discussed those matters which can be considered independentiy of a background gas or plasma. For some environments of envisioned application, and almost certainly for possible laboratory experiments, interaction of plasmoid particles with a background neutral gas may lead to important efforts. A neutral gas is singled out for consideration here, since this is expected to be the predominant component in situations of interest and to give rise to phenomena not readily treated by the methods discussed previously. In the AFWL report ${ }^{16}$ prepared by Calspan, Inc., for example, it is noted that the differential ionization energy loss suffered by electrons and ions, taken with their very different masses, leads to a "velocity slip" between the positive and negative components of the plasmoid. Further, the energy loss of the charged plasmoid particles, though negligible compared to the translational kinetic energy of the plasmoid particles can be comparable to or larger than the initial plasmoid thermal energy. Finally, CALSPAN notes that the velocity slip will give rise to an axial current and a corresponding azimuthal magnetic field.

At least one distinctly unrealistic feature renders the results of these calculations of dubious value. No attempt was made to include the electrostatic restorative forces which surely reduce such velocity slippage. Rather, these are invoked to mediate in some mysterious fashion the randomization of the kinetic energy difference thereby acquired. Very high resultant temperatures (20-100 ev) were thereby inputed to the plasmoid at the end of even modest flight distances. Further, it is not clear that the small number of effective collisions encountered under exoatmospheric conditions justifies the fundamental statistical assumptions which underlie the usual ionization energyloss formulae.

Nevertheless, an attempt has been made to formulace an approach to problems in which background gas interactions with plasmoid particles may play an important role. The principal cumplicating feature exhibited by this problam is that both the discrete, collisional elements and the collective 
aspects should be treated simultaneously and self-consistently. Generally, numerical models focus on one or the other aspect of these problems. No code is available which can treat simultaneously all relevant phenomena. Therefore, one must resort to an interactive application of at least two different sorts of numericul code. Such an interactive application is depicted in Figs. 2 and 3 , and the corresponding reinforcing flow of information which should characterize this approach is indicated in Fig. 4. Basically, one will empioy a Monte Carlo transport code to yield quantitative information concerning the effect of discrete interactions with background gas atoms. Such a calculation might generally be regarded as microscopic since collisions are occurring on an atomic scale. Sirce in this report the term microscopic refers more specifically to phase space-dependent collective phenomena', and the Monte Carlo calculations exclude such collective effects, we have included these calculations in the heuristic portion of our conceptual plan, Fig. 2. By running the Monte Carlo code with a variety of initial conditions, one may learn at least qualitatively what effect the background interactions have on the plasmoid distribution function. The particular Monte Carlo program which is being contemplated for use in the present study includes the effect of an externally prescribed, but not self-consistent, electromagnetic field on the trajectories of the particles. Thus, at least some of the effects of an external magnetic field, of particular interest for exoatmospheric plasmoid propagation matters, can be included in the calculation. Since Monte Carlo calculations are usually not effected over the distances likely to be of practical interest, some scalings may have to be made with respect to, for example, background density and magnetic field. Such scalings are also likely tu be of interest in relation to experimental, laboratory matters.

Data thus acquired from Monte Carlo calculations would then be employed to initialize the PIC simulation code. The objective of simulations performed with such an initialization would be to evaluate the effect of the collective, restorative forces neglected in the Monte Carlo calculations. For example, it has been suggested ${ }^{16,36}$ that tie velocity slippage between the two species will give rise to a macroscopic separation of charge and corresponding oscillations when the resultant electrostatic forces attempt to remove the separation. Such macroscopic oscillations could prove deleterious from the perspective of propagation. These arguments appear questionable, due to the small number of collisions expected to occur over contemplated flight distances. Nevertheless, the 
procedure described here should serve to resolve these issues. of course, one must throughout be guided by a certain amount of common sense and physical intuition. Monte Carlo results are likely to indicate velocity slippages and charge separations which are unrealistic when viewed in the context of a completely self-consistent calculation. Guided by such common sense and physical intuition, however, at least the principal effects of background interaction on the collective properties of plasmoid propagation should yield to analysis. We note also that, quite apart from problems arising from possible velocity slippage, changes in plasmoid particle distribution functions induced by background interactions may have an important effect on those microscopic collective processes which generate anomalous resistivity. All these questions may well prove to be more relevant to the planning and interpretation of scalable, labocatory experiments than to evaluation of performance in envisioned environments of operation.

Several times throughout the description of the suggested plan of study depicted schematically in Fig. 2, we have emphasized that the order of tasks appearing there is meant to be more conceptual than chronological. Both heuristic and nicroscopic considerations are expected to play a role throughout. Such an interleaving of calculations yields in a timely fashion important information which will be refined as the study progresses. Naturally, emphasis will be placed initially on those heuristic calculations designed to delineate the gross features of plasmoid propagation which arise independently of any background interactions. However, microscopic calculations could commence fairly soon after the first fluid determination of gross charge and current distributions, even though such simulations would lack the refinement accorded by the complete determination of particle orbits and the accompanying linear stability analysis. For instance, if early magnetohydrodynamic calculations indicated the presence of strong shearing motions within the plasmoid, particlein-cell simulation could commence even in the absence of a complete, kinetic, geometrically-realistic linearized stability theory. Any required numerical quantities needed to initialize such preliminary simulation could be taken, for example, from one of the fluid calculations effected in an idealized geometry which is available in the literature. ${ }^{37}$ Although such a preliminary simulation would not yield accurate transport coefficients, it might nevertheless serve to further define the course of the study. Indeed, it is through such measures that the detailed course of the study will. be determined dynamically. 
A rough order of tasks to be accomplished and the priorities to be assigned to them is nevertheless discernable. In fact, this order approximately corresponds to the conceptual sequence in Fig. 2 with, however, some importan: exceptions. Generally, we would concentrate on those questions involving the collective aspects of plasmoid propagation which do not entail interactions with background gas. Our reason for this selection is that it is the collective phenomena which are likely to prove the most deleterious from the point of view of propagation. Characterization of major areas of concern should clearly be a primary goal. Further, simple analytic considerations indicate that in environments of potential application the plasmoid-background gas interactions should play a strictly secondary role. Initial emphasis will naturally be placed on the heuristic aspects of the collective behavior of the plasmoid, with microscopic simulations following soon thereafter. No sharp chronological distinctions can be made in the heuristic-microscopic interplay, the process being more iterative than sequential in nature. Only after a fairly complete understanding of the collective features of plasmoid propagation in the absence of background interactions has been attained will an effort be made to include modifications due to a background gas. Knowledge of all these factors will be used to assess the effectiveness of various confinement schemes. At the conclusion of this study, one should have a complete view of the limiations to effective propagation of neutral plasma beans in parameter regimes of likely military interest.

\section{PEKSONNEL AND COMPUTATION RESOURCES}

In the preceeding section we have described a plan of investigation of neutral plasma beam propagation which makes extensive use of those concepts and computational methods long employed in the study of physical phenomena in which collective plasma effects play a dominant role. These concepts and methods have, in particular, been applied in recent years to analyze the physics of intense particle beams. Prerequisite to successful execution of the recommended plan is the availability of personnel with appropriate experience who have access to the necessary computational tools. In this section we very briefly describe such personnel and computational resources currently available at the Los Alamos Scientific Laboratory.

Group T-15, Intense Particle Beam Theory, is concerned solely with the investigation of the basic physics and possible applications of high-current, 
high-energy electron and ion beams. Present activities include investigations of collective ion acceleration schemes, plasma heating methods, propagation matters, free electron lasers, and a light-ion fusion concept. Emphasis is placed on the collective effects which dominate the dynamics of such systems. Problems encountered generally involve a combination of intense self-fields, complex geometries, explicit time dependence, and strong nonlinearity. Any one of these factors can render either difficult or impossible the application of the classical, analytic methods of theoretical physics. Hence, strong emphasis is placed upon the effective utilization of computational numerical techniques and tools. Various members of Group $\mathrm{T}-15$ have broad experience in the development and application of such tools. Al.though members of the group generally concentrate on one particular project, insights gained, experience acquired and numerical technqiues and tools developed are automatically available for application to any of the other problems being simultaneously pursued. Thus, any one particular problem is likely to benefit from activities being pursued in separate but related projects.

The principal numerical code employed in the investigation of intense particle beams at the Los Alamos Scientific Laboratory is CCUBE, a two-dimensional, relativistic, fully electromagnetic particle-in-cell simulation code developed by B. Godfrey. CCUBE may treat problems in any orthogonal curvilinear coordinate system, thus making possible the simulation of many realistic, geometrically complex systems. This is potentially an important point for plasmoid propagation studies. Novel numerical plasma simulation techniques, developed by Godfrey, ${ }^{38}$ building on ear?ier work of Lewis, ${ }^{39}$ are incorporated in this code. This code has been used in a variety of applications, including study of collective ion acceleration schemes, ${ }^{40}$ plasma heating investigations, 41 and the very preliminary simulation of the heuristic aspects of plasmoid propagation to be described in the next section.

Heuristic, fluid calculations can be effected with magnetohydrodynamic codes, several of which are available at the Los Alamos Scientific Laboratory, where they are employed in a host of projects. Two seem to be particularly usesul for plasmoid propagation studies. A two-dimensional, Eulerian code 19 developed by I. Lindemuth, formerly of the Lawrence Livermore Laboratory and presently at the Los Alamos Scientific Laboratory, appears to be the most useful numerical tool currently available for these purposes. The dynamical heart of this code, as with all other magetohydrodynamic codes, is the set of 
fluid equations expressing mass, monentum, and energy conservation. Features which make this computational tool particularly useful :For plasmoid propagation studies are the inclusion of a phenomological resistivity, energy loss due to bremsstrahlung and line radiation, and a moving frame option. Alternatingdifference: implicit methods are employed, making possible a larger time step than with explicit differencing schemes. Previous problems addressed with this code include Rayleigh-Taylor instabilities of a laser produced plasma decelerated by a magnetic field, 42 and dynamic theta pinch phenomena. ${ }^{43}$

A second magnetohydrodynamic code, developed by $\mathrm{J}$. Brackbill, 44 is available. This two-dimensional Lagrangian code is not as potentially useful for plasmoid propagation matters as the Eulerian code described above, principally because Lagrangian methods do not treat in an effective fashion the strong shearing motion so characteristic of many magnetohydrodynamic flows. However, there remains the possibility that problems will arise in which fluid-vacuum and fluid-fluid interfaces must be treated very accurately. For such detailed computations a Lagrangian approach is frequently preferrable. Although not as many physics options are available as is the case with the Eulerian code, a phenomological resistivity is included in the equations of motion. Published applications include the study of a laser produced plasma expanding across a magnetic field and the evolution of a pinched plasma.

Matters pertaining to interaction of charged particles with a neutral background gas are usually addressed through Monte Carlo transport techniques. 45 A particularly useful embodiment of these techniques exists at the LASL in the form of the code CYLTRAN. ${ }^{46}$ The present version of this code computes electron, ion and photon transport consistently with the presence of an external prescribed electromagnetic field. This code has been applied previously, ${ }^{46}$ for example, to the calibration of charged particle counting devices and the transport of relativistic electron beams in an environment corresponding to that of an inertial confinement fusion scheme.

As emphasized previously, problems being pursued by Group T-15 members generally benefit from activities related to other projects. Study of plasmoid propagation across a magnetic field would be no exception. At least two numerical codes likely to be developed during the next year and a half will be of either direct or indirect interest for plasmoid propagation. One such code is a three-dimensional version of the two-dimensional PIC code CCUBE described earlier. The practicality of developing and using such a numerical tool is 
considerably enhanced by the availability of a Cray-1 computer at Los Alamos and the imminent acquisition of another such machine. Although, as we have seen, numerous quesfions of interest for plasmoid propagation can be answered with the presently available two-dimensional version, there are several questions which could benefit immensely from three-dimensional simulations. These include matters related to the influence of boundaries on current flow and the three-dimensional character of possible confining forces. Due to the possibly complicated nature of particle orbits in a finite propagating plasmoid, the anomalous resistivity-generating microinstabilities may also have distinct three-dimensional characteristics. This code will probably not be available until the middle or latter stages of the suggested study; and we have, therefore, not made it an integral part of our prospective computational arsenal. However, it is well to bear in mind that availability at that time may offer a unique opportunity to assess the validity of earlier geometric simplifications and idealizations necessitated by requirements of analytic tractability or computational limitations.

In our recommended plan of study, we have emphasized the desirability of obtaining a kinetic, geometrically realistic linearized theory of the microinstabilities expected to generate anomalous transport coefficients. Some members of Group T-15 are currently interested in developing a finite-temperacure kinetic version of a code previously written and applied by B. Godfrey 47 to the linear stability analysis of radially inhomogeneous nonneutral, relativistic electron beams. Such a code will probably involve explicit integrations along unperturbed particle orbits. Although the geometry and self-fields for this particular problem will no doubt be different from those encountered in plasmoid propagation, the techniques developed will probably have a much wider applicability embracing questions of present interest. Other, similar but unanticipated advantages for plasmoid propagation matters are likely to accrue during the course of the recommended study.

\section{INITIAL RESULTS}

Several times during the course of this report, we have referred to a simple qualitative picture of the inicial stages of plasmoid propagation. According to this picture, a sufficiently energetic plasmoid will propagate by initially excluding the ambient magnetic field from its interior through the establishement of diamagnetic currents. In order to gain confidence in this 
picture, and to obtain some understanding of its limitations, we have performed a PIC simulation, using the numerical code CCUBE described earlier, of the very early stages of propagation. A further, perhaps equally important, purpose of this simulation is to provide a sampling of the varied kinds of information obtainable from such a code. Since the plasmoid is simulated as a whole, the simulation is in particular an example of an heuristic application of CCUBE, as discussed in Section IV. Since this simulation is a very preliminary exercise, several aspects were hardly optimized to yield the quality of information and insight to be expected from a simulation effected during the course of a systematic study, such as that depicted in Section IV. Nor have the results of the simulation been subjected to any extensive analytic investigation and comparison, an equally vital aspect of a full program. Nevertheless, these admittedly very preliminary results are themselves of some interest and provide some hint of what would be obtained from at least one phase of the recommended program. This brief section is devoted to a description of this simulation. In the simulation, a neutral plasma beam was injected at the left boundary of a computational region which was permeated by a magnetic field initially pointing in the direction perpendicular to the direction of propagation of the plasmoid. The plasmoid propagated the length of the computational box, exiting from the right boundary. Time of simulation was, therefore, limited by the plasmoid time of transit. As discussed previously, this limitation would be removed at the start of a full study by transforming to the plasmoid frame, or perhaps by adding a moving frame option. For plasmoid parameters, we chose values relevant to the TRW, Inc., proposal. 17 The physical dimensions of the plasmoid were chosen differently from the TRW specifications to facilitate fitting the system into the computational mesh without loss of desired resolution. The ratio of these dimensions was maintained, however. Since we are here simulating cross-field propagation with a two-dimentional code, we employ a rectangular coordinate system. Thus, the cross section of the plasmoid to be displayed is rectangular, rather than cylindrical. Desire to simulate crossfield propagation of a truly cylindrical plasmoid, corresponding to the anticipated experimental system, is one of the motivating factors in the creation of the three-dimensional PIC code referred to in the previous section. The ambient magnetic field was chosen to be 0.35 gauss, corresponding roughly to the magnitude of the geomagnetic field at ionospheric altitudes. A realistic mass ratio of 1836 was employed in keeping with our intentions of examining 
heuristic aspects of propagation whose features are determined at least qualitatively by the ratio of kinetic to field energy densities. As a consequence, however, nost of the dynamical features of the simulation are due to electron, rather than ion, response.

CCUBE usually generates a large number of particle and field diagnostic plots which yield a great variety of detailed dynamical information. It is clearly impractical to display them all here. Our selection of those to exhibit was guided by our desire to demonstrate the most basic features of field exclusion. To exhibit these as clearly as possible, we have chosen three plots from three different times during the course of the simulation. These three sets of three graphs are displayed successively in Figs. 5, 6, and 7 . The corresponding times were $t=40 \mathrm{w}_{\mathrm{p}}^{-1}, 120 \mathrm{w}_{\mathrm{p}}^{-1}$, and $180 \mathrm{w}_{\mathrm{p}}^{-1}$ to represent typical field and current configurations at times soon after injection, during the principal phase of the simulation, and immediately before plasmoid exit from the computational box. $w_{p}^{-1}$ is here the vibration period for an electron collective oscillation and is the time urit natural to the numerical code. For the TRW, Inc., figures ${ }^{17}$ this corresponds to approximately a tenth of a nanosecond. Usually, the unit of length employed is $c / w_{p}$, where " $c$ " is the velocity of light. For convenience in interpretation of the figures, however, we have initialized the code such that this quantity is equal to a centimeter. Magnetic fields are expressed in terms of cyclotron frequencies normalized to that corresponding to the initial external magnetic field.

First in each of three sets of three graphs is simply a plot of the plasmoid particle positions, which serves to locate the plasmoid within the computational box. Second in each set of three is a contour plot of that magnetic field component pointing in the direction of the initial, undisturbed external magnetic field. Darker lines indicate stronger fields. Lastly, we exhibit a plot of the current perpendicular to the plane of calculation versus the coordinate for the direction in which the plasmoid is propagating. Although the significance of the first two plots may be obvious, the selection of the third from other interesting diagnostic plots perhaps requires some explanation.

As the plasmoid enters the region of magnetic field, the plasma particles experience a Lorentz $V \times B$ force. Since the electrons are so much lighter, they respond much more readily to this force. Subsequently, they establish a current in the direction perpendicular to the plane of direction of plasmoid propagation and the initial magnetic field. In the case of our figures, this, 
of course, corresponds to the direction of the z-axis. The magnetic field produced by this current tends to decrease the magnetic field imediately inside the leading edge of the plasmoid, and to slightly increase it imediately outside. Thus, we expect to see the magnetic field contours billow out in front of the plasmoid and to be excluded from its interior. Since it is the magnetic field which initially establishes the current, one anticipates the current to decrease as one progresses into the plasmoid from the leading edge. This justifies cur selection of the $J_{z}$ current density plot. Such a simple physical picture underlies the work of Ferraro, ${ }^{3}$ and it is to illustrate this picture and to delineate some of its limitations that we are presenting the accompanying diagrams.

Examination of Figs. 5-7 shows that many qualitative features of this description are indeed verified by the simulation, but with some major quantitative differences. The magnetic field is indeed pushed out in front of the plasmoid and decreased in the interior. However, at no point does the field seem to be completely excluded, the magnitude of the $B_{y}$ component decreasing by, at most, a factor of ten. Further, the field extends into the plasmoid much further than the collisionless skin depth as predicted by Ferraro. Arditional departures from our simple description and some insight into their origin can be gained by examining the $J_{z}-x$ plots. The various curves exhibited there represent $x$-profiles taken at selected values of constant $y$. Those which are most sharply peaked and fall. fastest into the plasmoid are taken from longitudinal cuts near the center of the plasmoid, while the broader curves represent currents flowing on the upper and lower surfaces of the plasmoid. These are naturally expected to persist. since they are flowing on or near a vacuum-plasmoid interface. This finite geometry effect is an apparently important element for the interpretation of this simulation which is not included in Ferraro's ${ }^{3}$ analysis and a good example of the variety phenomena which would emerge from a complete, systematic study of plasmoid propagation.

Much further analysis is required to come to a complete understanding of even this preliminary simulation. A logical way in which to begin such further analysis might be by appropriately generalizing Ferraro's ${ }^{3}$ analysis to include at least some finite geometry effects. Even simple current sheet models may prove useful in this regard. Examination of other diagnostics provided by this simulation indicate the initial stages of unexpected, and at this point unexplained, collective phenomena. $I^{+}$should perhaps be noted that finite rise-time 
and numerical discreteness effects may also be contributing to the incomplete exclusion of the magnetic field from the plasmoid. Further simulations, of a relatively short and inexpensive nature, could throw much additional light on these questions. One could, for example, perform a simulation with different physical plasmoid dimensions to examine finite-geometry effects, and optimize various plotting parameters to glean more information from the particle diagnostics. All these tasks would receive high priority during the initial phase of a complete systematic study of neutralized plasma beam propagation across a magnetic field.

\section{ACKNOWLEDGMENTS}

During the preparation of this report, the authors benefited from discussions with numerous colleagues. Particular thanks are extended to P. Gary, J. Kindel, I. Lindemuth, E. Lindman, J. Mack, and J. Marshall, all of the Los Alamos Scientific Laboratory, Z. G. T. Guiragossian of TRW, Inc., W. Thompson and G. Gullespie of Physical Dynamics, Inc., and C. Longmire of Missions Research, Inc.

\section{REFERENCES}

1. S. Chapman and V. C. A. Ferraro, "A New Theory of Magnetic Storms," Terr. Mag. (J. Geophys. Res.) 36, 77 (1931).

2. E. 0. Hulbert, "Terrestial Magnetic Variations and Aurora," Revs. Mod. Phys. 8, 67 (1937).

3. V. C. A. Ferraro, "On the Theory of the First Phase of a Geomagnetic Storm: A New Illustrative Calculation Based on an Idealized (Plane Not Cylindrical) Model Field Distribution," J. Geophys. Res. 57, 15 (1952).

4. D. M. Wetstone, M. P. Ehrlich, and D. Finkelstein, "Plasmoid Motions Along Curved Magnetic Field Lines," Phys. Fluids 3 , 617 (1960).

5. G. Schmidt, "Plasma Motion Across Magnetic Fields," Phys. Fluids 3, 961 (1960).

6. D. A. Baker and J. E. Hammel, "Experimental Studies of the Penetration of a Plasma Stream in a Transverse Magnetic Field," Phys. Fluids $\underline{8}, 713$ (1965).

7. A. Komori, N. Sato, H. Sugai, and Y. Hata, "Drift Motion of a Plasma in a Curved Magnetic Field," Plasma Phys. 19, 283 (1977). 
8. W. Bostick, "Experimental Study of Ionized Matter Projected Across a Magnetic Field," Phys. Rev. 104, 292 (1956).

9. W.Bostick, "Experimental Study of Plasmoids," Phys. Rev. 106, 404 (1957).

10. S. Chandrasekhar and E. Fermi, "Problems of Gravitational Stability in the Presence of a Magnetic Field," Astrophys. J. 118, 116 (1953).

11. G. Schmidt, "Virial Theorem for Plasmas," Phys. Fluids $\underline{3}, 481$ (1960).

12. R. B. R. Shersby-Harvie, "Schmidt's Virial Theorem for a Plasma," Phys. Fluids 4 , 389 (1961).

13. E. Ott and W. Manheimer, "Cross-field Injection, Propagation, and Energy Deposition of Intense Ion Beams with Application to Tokamak Plasma Heating," Nuclear Fusion 17, 1057 (1.977).

14. H. Grad, "Boundary Layer Between a Plasma and a Magnetic Field," Phys. Fluids 4, 1366 (1961).

15. S. Jorna and W. B. Thompson, "Beam Stability," Physical Dynamics, Inc. report PDL-7660 (1976).

16. CALSPAN Corporation, "Theoretical Analysis of Neutralized Beam Propagation," Air Force Weapons Laboratory report AFWL-TR-78-52 (1979).

17. 2. G. T. Guiragossian of TRW, Inc. (private communication). The relevant parameters for this proposed scheme are $n_{p}=6.5 \times 10^{10}$ and $v_{p} / c=0.42$.

18. L. Spitzer, Physics of Fully Ionized Gases, Interscience Tracts on Physics and Astronomy, 3, Interscience Publishers (1962).

10. I. R. Lindemuth, "The ANIMAL Code," Lawrence Livermore Laboratory report UCRL-52492 (1979).

20. B. B. Godfrey, "The Localized Pinch Model as a High Energy Ion Collective Acceleration Mechanism," IEEE Trans. Plasma Sci. PS-6, 256 (1.178).

21. R. L. Morse, in Methods in Computational Physics, B. Adler, S. Fernbach, and M. Rotenberg, eds., Vo1. 9 (1969).

22. S. Chandrasekhar, "Stochastic Problems in Physics and Astronomy," Revs. Mod. Phys. 15, 1 (1944).

23. D. Bohm, The Characteristics of Electrical Discharges in Magnetic Fields, A. Guthrie and R. K. Wakerling, eds. (McGraw-Hill, New York, 1949) Chapter 2, Section 5.

24. L. Spitzer, "Particle Diffusion Across a Magnetic Field," Phys. Fluids $\underline{3}$, 659 (1960).

25. W. Drummond and M. Rosenbluth, "Anomalous Diffusion Arising from Microinstabilities in a Plasma," Phys. Fluids $\underline{5}, 1507$ (1962). 
26. C. Oberman and A. Rogister, "On the Kinetic Theory of Stable and Weakly Unstable Plasmas," J. Plasma Phys. 2, 33 (1968).

27. 0. Buneman, "Dissipation of Currents in Ionized Media," Phys. Rev. 115, 503 (1959).

28. B. Fried and R. Gould, "Longitudinal Ion Oscillations in a Hot Plasma," Phys. Fluids $\underline{4}, 139$ (1961).

29. C. T. Dum, R. Chodura, and D. Biskamp, "Turbulent Heating and Quenching of the Ion Sound Instability," Phys. Rev. Lett. 32, 1231 (1974).

30. R. Sagdeev, "The 1976 Oppenheimer Lectures: Critical Problems in a Plasma Astrophysics I. Turbulence and Nonlinear Waves," Revs. Mod. Phys. 51, 1 (1979).

31. A Hirose and I. Alexeff, "Anomalously Rapid Skin-Current Penetration and Heating in Pulsed Plasmas," Phys. Rev. Lett. 26, 949 (1969).

32. C. Longmire, Elementary Plasma Physics Monographs and Texts in Physics and Astronomy (Interscience Publishers, New York, 1962), Chapter XI, and many references cited tierein.

33. N. Krall, "Drift Waves," in Advances in Plasma Physics, Vol. 1, A. Simon and $W$. Thompson, eds. (Interscience Publishers, New York, 1968).

34. T. W. Speiser, "Particle Trajectories in Model Current Sheets," J. Geophys. Res. 70, 4219 (1965).

35. H. Okuda and C. K. Birdsa11, "Collisions in a Plasma of Finite-Size Particles," Phys. Fluids 13, 2123 (1970).

36. 2. G. T. Guiragossian (private communication).

37. E. R. Harrison, "Longitudinal Electrostatic Oscillations in VelocityGradient Plasmas. I. The Slipping-Stream Mode1," Proc. Phys. Soc. 82, 685 (1963).

38. B. Godfrey, "A Galerkin Algorithm for Multidimensional Plasma Simulation Codes," Los Alamos Scientific Laboratory report LA-7687 (1979).

39. H. R. Lewis, "Applications of Hamilton's Principle to the Numerical Analysis of Vlasov Plasmas," in Methods in Computational Physics, Vol. 9, B. Adler, S. Fernbach, and M. Rotenberg, eds. (Academic Press, Inc. 1970).

40. B. B. Godfrey, R. J. Faeh1, B. S. Newberger, W. R. Shanahan, and I. E. Thode, "Collective Ion Acceleration, July 1976 - September 1977," Los Alamos Scientific Laboratory report LA-7148-PR (1978).

41. L. Thode, "Plasma Heating by Scattered Relativistic Electron Beams," Phys. Fluids 19, 831 (1976). 
42. J. Allbriton, J. Cohen, R. Devoto, and R. Rowlands, "Flute Instability of a Laser-Pellet Plasma in a Magnetic Mirror," in Proceedings of Annual Meeting on Theoretical Aspects of Controlled Thermonuclear Fusion, Madison, Wisconsin, April 1976.

43. I. Lindemuth and J. Killern, "Alternating Difference Implicit Techniques for Two-Dimensional Magnetohydrodynamic Calculations," J. Comp. Phys. 13, 181 (1971).

44. J. U. Brackbill and W. E. Pracht, "An Implicit Almost-Lagrangian Algorithm for Magnetohydrodynamics," J. Comp. Phys. 13, 455 (1971).

45. M. J. Burger, "Monte Carlo Calculation of the Penetration and Diffusion of Charged Particles" in Methods in Computational Physics, Vol. 1, B. Adler, S. Fernbach, and M. Rotenberg, eds. (Academic Press, New York, 1963).

46. J. A. Halbleib, Sr., and W. H. Vandevender, "Coupled Electron Photon Collisional Transport in Externally Applied, Electromagnetic Fields," J. App. Phys. 48, 231 (1977).

47. B. B. Godfrey, "Linear Theory of the Radially Inhomogeneous Unneutralized Relativistic Electron Beam," IEEE Trans. Plasma Sci. PS-7, 53 (1979).

Controlled Thermonuclear Injection

Experiment Plasma

$\sim 10^{-2}$

Quiet-day Solar Wind in Vicinity

of Earth $\left(10^{-4}\right.$ gauss)

$\sim 1$

TRW, Inc, Military Applications

Neutral Plasma Beam Proposal

$$
\sim 10^{10}
$$

Fig. 1. A comparison for three different plasma systems of the magnitude of a quantity measuring the importance of diamagnetic effects reveals that the parameter regimes of military interest are much different from those of previous studies. 


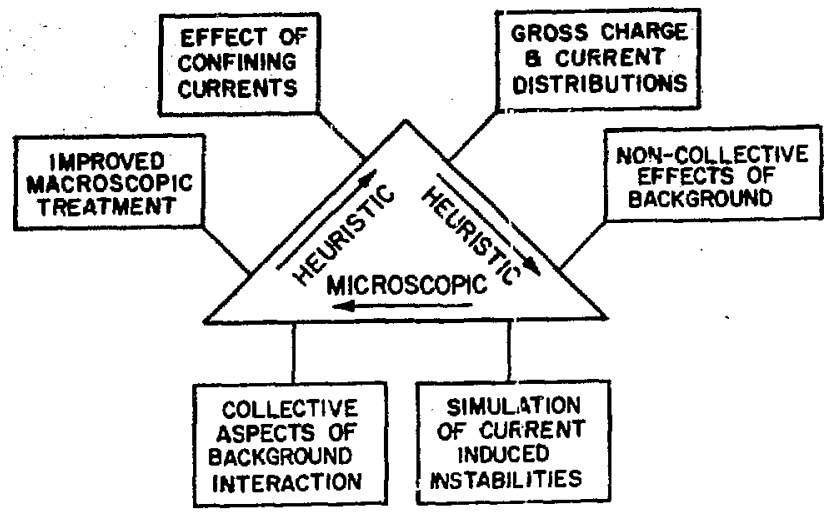

Fig. 2.

A program combining heuristic, macroscopic modelling with microscopic, particle-in-cell simulations can answer important questions concerning plasmoid propagation.

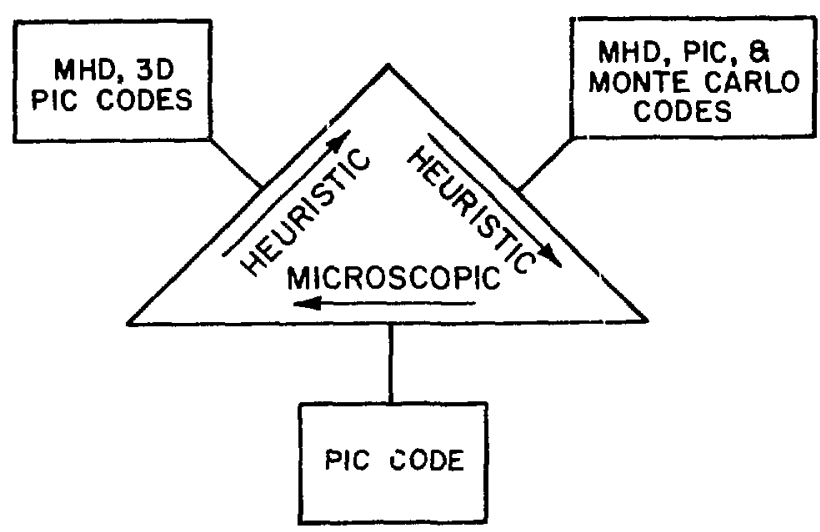

Fig. 3.

A variety of computational tools would be employed in the program.

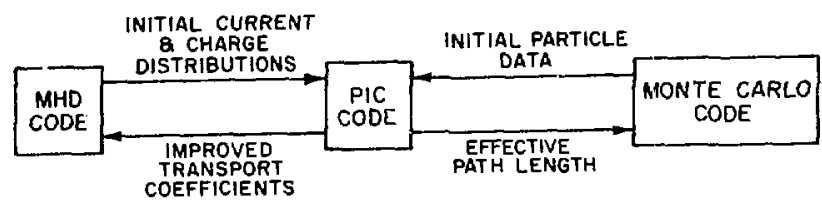

Fig. 4 .

A reinforcing flow of information between the heuristic modelling and the microscopic, particle-in-cell simulations characterizes the recommended program.
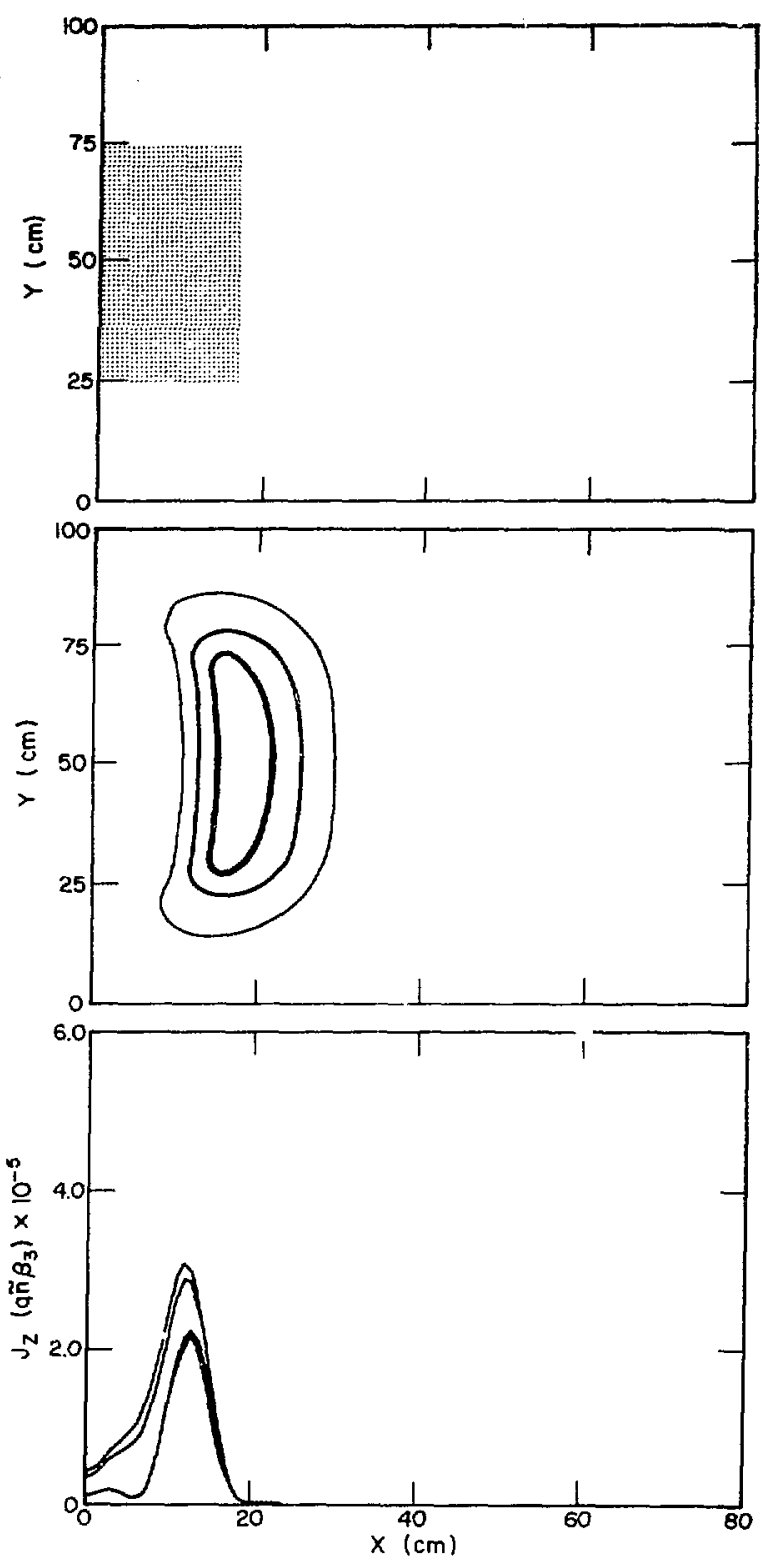

Fig. 5 .

Three plots taken from the early phase $\left(\omega_{p} t=40\right)$ of a computer simulation of plasmoid propagation across a magnetic field. First plot shows location of plasmoid in computational box. Second plot exhibits magnetic field contours, with darker lines corresponding to greater field strengths. Jast panel shows $x$-plots of current density for various $y$-slices. Those for which the current density is localized at the front of the plasmoid are taken from near the transverse middle, while those that extend backward represent surface effects. 

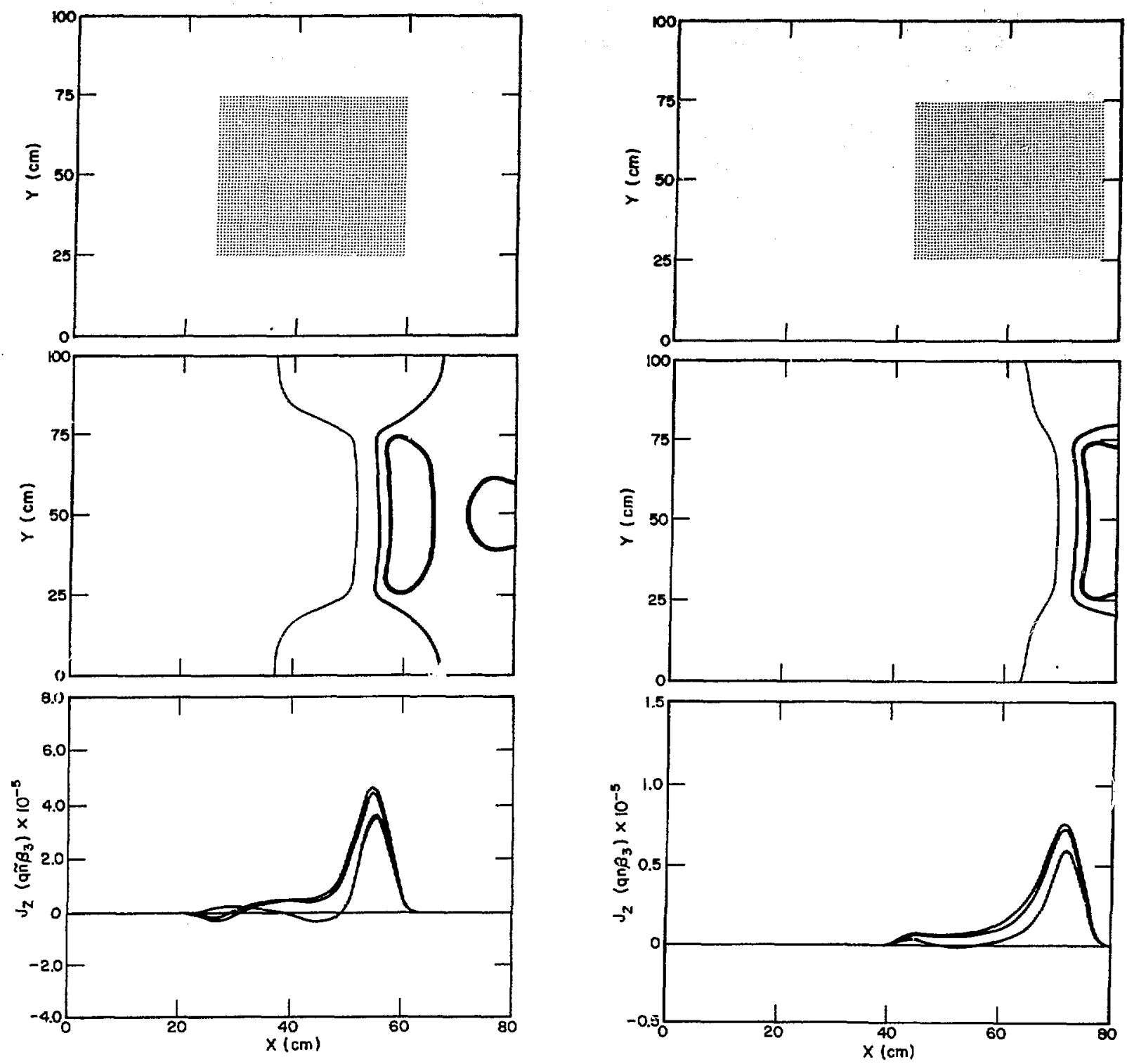

Fig. 6.

Fig. 7.

Same as Fig. 5 except $\left(\omega_{p} t=120\right)$.

Same as Fig. 5 except $\left(\omega_{p} t=180\right)$. 